\title{
Exploring Modality Compatibility in the Response-Effect Compatibility Paradigm
}

\author{
Noémi Földes ', Andrea M. Philipp ', Arnaud Badets' ${ }^{2}$, and Iring Koch' \\ 'RWTH Aachen University, Aachen, Germany \\ ${ }^{2}$ CNRS, Institut de Neurosciences Cognitives et Intégratives d'Aquitaine (UMR 5287), Université de Bordeaux, France
}

\section{KEYWORDS}

action control, ideomotor principle, response-effect compatibility, modality compatibility
ABSTRACT

\begin{abstract}
According to ideomotor theory, action planning is based on anticipatory perceptual representations of action-effects. This aspect of action control has been investigated in studies using the response-effect compatibility (REC) paradigm, in which responses have been shown to be facilitated if ensuing perceptual effects share codes with the response based on dimensional overlap (i.e., REC). Additionally, according to the notion of ideomotor compatibility, certain response-effect (R-E) mappings will be stronger than others because some response features resemble the anticipated sensory response effects more strongly than others (e.g., since vocal responses usually produce auditory effects, an auditory stimulus should be anticipated in a stronger manner following vocal responses rather than following manual responses). Yet, systematic research on this matter is lacking. In the present study, two REC experiments aimed to explore the influence of R-E modality mappings. In Experiment 1, vocal number word responses produced visual effects on the screen (digits vs. number words; i.e., visual-symbolic vs. visual-verbal effect codes). The REC effect was only marginally larger for visual-verbal than for visual-symbolic effects. Using verbal effect codes in Experiment 2, we found that the REC effect was larger with auditory-verbal R-E mapping than with visual-verbal R-E mapping. Overall, the findings support the hypothesis of a role of R-E modality mappings in REC effects, suggesting both further evidence for ideomotor accounts as well as code-specific and modality-specific contributions to effect anticipation.
\end{abstract}

\section{INTRODUCTION}

The ideomotor principle states that an anticipatory perceptual representation is activated before every voluntary action (e.g., Greenwald, 1970). For example, when turning on the light, one anticipates perceptual consequences such as receiving more light and feeling a little pressure under the fingertips as they press the light switch. The activation of this perceptual anticipation is thought to be the cognitive basis for voluntary action execution (e.g., Ansorge, 2002).

In some studies, the notion of effect anticipation was examined with the response-effect (R-E) learning paradigm, in which it could be shown that merely displaying any kind of effect after the response and then using the effect later as stimulus for the previously associated response leads to facilitation relative to using a stimulus that was previously associated with a different response (e.g., Badets \& Pesenti,
2011; Elsner \& Hommel, 2001; Herwig, Prinz, \& Waszak, 2007; Herwig \& Waszak, 2009; Hommel, 2013; Hommel, Alonso, \& Fuentes, 2003; Janczyk, Heinemann, \& Pfister, 2012; Pfister, Kiesel, \& Hoffmann, 2011; Ziessler, Nattkemper, \& Frensch, 2004). Thus, this paradigm requires an R-E learning phase and an additional transfer test phase where the effect is used as stimulus.

In contrast to the R-E learning paradigm, the response-effect compatibility (REC) paradigm does not require an initial learning phase because it exploits preexisting relations (e.g., spatial) between respons-

Corresponding author: Noémi Földes, Institute of Psychology, RWTH Aachen University, Jägerstraße 17-19, 52066 Aachen, Germany.

E-mail: Noemi.Foeldes@psych.rwth-aachen.de 
es and effects arising from dimensional overlap between responses and their predictable effects (see Kornblum, Hasbroucq, \& Osman, 1990, for the dimensional overlap account).

\section{Effect Anticipation in the Response-Effect Compatibility Paradigm}

In the REC paradigm, the participant receives a response-contingent effect which is either compatible or incompatible with their response (e.g., Janczyk, Pfister, \& Kunde, 2012; Janczyk, Yamaguchi, Proctor, \& Pfister, 2015; Keller \& Koch, 2006; Koch, Keller, \& Prinz, 2004; Kunde, Koch, \& Hoffmann, 2004; Pfister, Kiesel, \& Melcher, 2010; Pfister \& Kunde, 2013; see also Badets, Koch, \& Philipp, 2016; Shin, Proctor, \& Capaldi, 2010, for reviews). For example, Kunde (2001, Experiment 2) instructed participants to press keys softly or forcefully, which then either led to a quiet or loud tone (manual-auditory R-E mapping). Whether a trial was compatible or incompatible was defined based on the intensity dimension (e.g., soft press and quiet tone was an R-E compatible mapping). Importantly, effects were fully predictable in a given block of trials, so that stable R-E anticipations could be formed. Kunde found an REC benefit (shorter response times [RTs] in the compatible condition), suggesting that anticipating a compatible effect facilitates, or primes, the corresponding responses.

In another study (Badets, Koch, \& Toussaint, 2013, Experiment 1), participants executed an R-E task where they saw a geometrical shape (a triangle or a square) as a stimulus to which they responded vocally with the number two or eight according to an instructed stimulusresponse (S-R) mapping. After their response, the digit 2 or 8 appeared on the screen (i.e., a vocal-visual R-E mapping) in one block in an R-E compatible way (e.g., 2 appears after the response two) and in another block in an R-E incompatible way ( 2 appears after the response eight). Results showed RTs shorter by $19 \mathrm{~ms}$ in the compatible condition compared to the incompatible condition, which, according to the authors, was due to the effects' magnitude representation (Walsh, 2003). These findings extend the REC paradigm to vocal tasks (see also Koch \& Kunde, 2002) as well as to numerical R-E compatibility relations.

\section{Modality Compatibility and Ideomotor Compatibility}

According to ideomotor theory, actions are coded in a mental representation based on the perceptual characteristics of the feedback (or effect) these actions create. Based on this notion, Greenwald (1972) assumed that since vocal responses (or actions) usually cause auditory feedback (hearing one's own voice while speaking or receiving an answer from the partner during verbal communication) and manual responses are often followed by visible effects (e.g., when pointing, writing, or turning on the light), the vocal-auditory and the manual-visual R-E modality mappings are stronger than the vocal-visual and manual-auditory mappings. Accordingly, stimuli that closely match the natural sensory effects should be particularly compatible (ideomotor compatibility; see Greenwald, 1972).
In contrast to the present R-E study, the notion of ideomotor compatibility has been mainly tested in S-R compatibility studies so far, using stimuli which strongly resemble the effect of the response that was required for the presented stimulus (note that stimuli in an S-R task and effects in an R-E task can be presented in the same way, see Figure 1). Specifically, the first study investigating ideomotor compatibility used dual-tasks where participants executed two simple spatially S-R compatible tasks in the same trial (Greenwald, 1972; Greenwald \& Shulman, 1973). Participants were presented with a visual and an auditory stimulus (an arrow pointing toward left or right, and the word left or right spoken in their headphones) and had to execute two responses (move a switch left or right and say the word left or right; Greenwald, 1972). Based on the instruction, participants performed either-using Greenwald's (1972) definitions-ideomotor compatible tasks (manual $\mathrm{R}$ to visual $\mathrm{S}$ and vocal $\mathrm{R}$ to auditory $\mathrm{S}$ ) or ideomotor incompatible tasks (manual $\mathrm{R}$ to auditory $\mathrm{S}$ and vocal $\mathrm{R}$ to visual $\mathrm{S}$ ), even though both were spatially S-R compatible, differing only in the S-R modality mappings. The study revealed lower dual-task costs when both tasks were ideomotor compatible (see also Greenwald, 2005; for a critical view see Lien, Proctor, \& Allen, 2002). Such effects of modality mappings have been replicated in dual-tasks (Göthe, Oberauer, \& Kliegl, 2016; Hazeltine, Ruthruff \& Remington, 2006).

Recently, this notion of ideomotor compatibility was reexamined as modality-specific compatibility in the context of a sequential task switching paradigm (Schäffner, Koch, \& Philipp, 2016; Stephan \& Koch, 2010, 2011, 2016). Stephan and Koch (2010) instructed participants to switch between two spatial S-R tasks. In the modality compatible condition, participants were instructed to respond by pressing the left or right arrow key on a keyboard to a visual stimulus presented either on the left or on the right side of the screen in one task, and to respond vocally by saying left or right to auditory stimuli presented to the left or the right ear in the other task. In the modality incompatible condi-

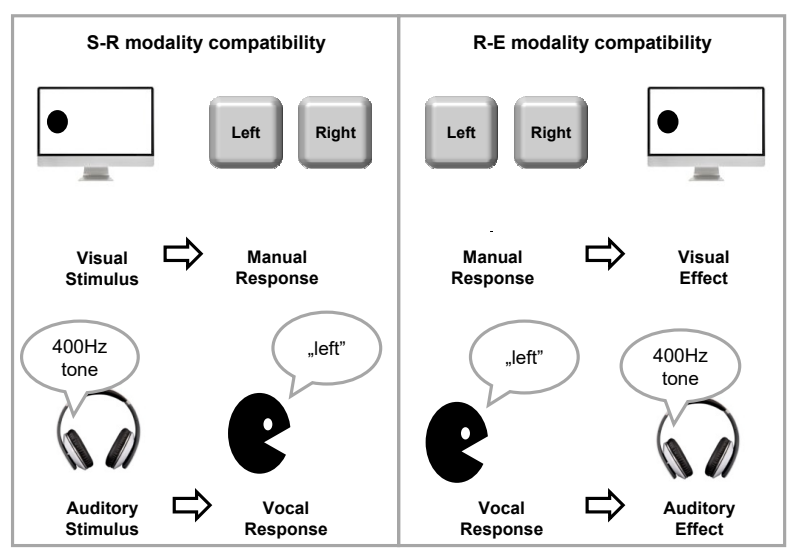

FIGURE 1.

An illustration of how a response-effect (R-E) task (i.e., output-input task) can be created from a stimulus-response (S-R) task (input-output task), reversing the order of procedure in the task in order to investigate modality compatibility (Greenwald, 1972; Stephan \& Koch, 2010, 2011, 2016). 
tion, the visual stimulus required a vocal response and the auditory stimulus a manual response. Thus, in this experiment, the same S-R modality mappings were considered compatible (visual-manual and auditory-vocal) and incompatible (visual-vocal and auditory-manual), as defined earlier by Greenwald (1972). Stephan and Koch (2010) found reduced switch costs when switching between tasks involving compatible modality mappings compared to switching between tasks with incompatible modality mappings, reflecting less between-task crosstalk when participants had to switch between two compatible mappings (see also Stephan \& Koch, 2016).

\section{Modality Compatibility and Code Compatibility}

Wickens, Sandry, and Vidulich (1983) suggested that, besides input and output modalities, an additional component should be included when investigating optimal task performance based on S-R compatibility. According to their suggestion, vocal responses usually involve verbal, linguistic content (i.e., for communicative purposes), while manual responses are mostly used to execute tasks with strong spatial aspects, and thus, these output modalities are strongly associated with these central processing codes. That is, even though it is possible to connect vocal responses with spatial codes (e.g., vocalizing to produce localization information for another person) or manual responses with verbal codes (e.g., hand-writing results in a written word), performance should be better with some combinations of central code and output modality (e.g., vocal-verbal) than with other combinations (e.g., vocal-spatial). Their findings from dual-task experiments (similarly to previous studies on S-R compatibility) suggest that beyond S-R compatibility, compatibility between central processing codes and inputoutput modalities plays a role in human performance (see also Göthe et al., 2016; Wickens \& Liu, 1988).

Thus, the present study takes into consideration a possible compatibility between central codes and the modality of vocal responses, and investigates whether they play a role in R-E compatibility.

\section{The Present Experiments}

The R-E task used in our experiments is based on Experiment 1 from Badets et al. (2013). Similarly to their procedure, in Experiment 1 of the present study, visual stimuli called for vocal responses, which were followed by a visual effect (i.e., a digit or a number word was presented, either R-E compatible or incompatible). That is, we used a modality incompatible R-E mapping, but we specifically varied the type of the central code of the effect, presenting it either with visual-symbolic (i.e., digit) or with visual-verbal (i.e., number word) code.

Effect codes were varied in a blocked (and therefore predictable) manner. Thus, we created R-E code compatible conditions (vocal response followed by an effect in the form of a written number word) and code incompatible conditions (vocal response followed by an effect in the form of an Arabic digit). In Wickens and Liu's (1988) view, performance in a task can be defined based on a model including input modality (visual or auditory), central codes (spatial or verbal), and output modality (manual or vocal, see also Göthe et al., 2016). Since, according to the model, interference will be reduced if the central code component corresponds with the output modality component (e.g., verbal code and vocal response, Wickens et al., 1983), we reasoned that a verbal effect (e.g., written number word format) should resemble the vocal number word response more than a visual Arabic digit (Göthe et al., 2016), thus causing a more pronounced REC effect. Thus, in Experiment 1, we used a modality incompatible R-E mapping (vocalvisual), but we varied the central code presentation of the effect. We term this type of compatibility $R$-E code compatibility. We expected to find a larger REC effect with number words rather than with digits, since the first shares the verbal central codes with the response while the latter automatically activates spatial attributes (e.g., small and large numbers are represented on a mental number line from the left to the right part of the space, respectively, Dehaene, Bossini, \& Giraux, 1993).

In Experiment 2, we used a similar design, but compared performance with modality compatible vocal-auditory R-E mapping with a modality incompatible vocal-visual mapping with written number words as effects. There is R-E code compatibility in both conditions (i.e., verbal codes for R and $\mathrm{E}$ ), but they differ in R-E modality mapping (vocal-auditory vs. vocal-visual). We expected to find a stronger REC effect when the mapping is modality compatible (i.e., vocal-auditory) than when it is incompatible (i.e., vocal-visual).

\section{EXPERIMENT 1}

\section{Method}

\section{PARTICIPANTS}

Twenty-four German-speaking students from RWTH Aachen University $\left(M_{\text {age }}=23\right.$ years; six males and 18 females $)$ participated. All participants gave written consent and received partial course credit or monetary compensation. Each one of them reported normal or corrected-to-normal vision and was unaware of the experimental goals.

\section{APPARATUS}

The apparatus consisted of a PC equipped with a 17 in. computer screen with a $75 \mathrm{~Hz}$ refresh rate and a microphone, both approximately $60 \mathrm{~cm}$ from the participant. The presented stimuli were a triangle with $11 \mathrm{~cm}$ on each side and a square with $10 \mathrm{~cm}$ on each side, with black outlines on white background. The number-word effects were $3.2 \mathrm{~cm}$ wide and $0.9 \mathrm{~cm}$ high in $80 \%$ of the trials (i.e., frequent trials, see the Procedure section), while in the remaining 20\% (deviant trials, see the Procedure section), they were $1.6 \mathrm{~cm}$ wide and $0.7 \mathrm{~cm}$ high (the word $z w e i$, German for two) and $1.5 \mathrm{~cm}$ wide and 0.7 high (the word acht, German for eight). The frequent digit effects were $0.8 \mathrm{~cm}$ wide and 1.2 $\mathrm{cm}$ high, while the deviant digit effects were $0.4 \mathrm{~cm}$ wide and $0.6 \mathrm{~cm}$ high. The effects were written in black color and with Arial font. 


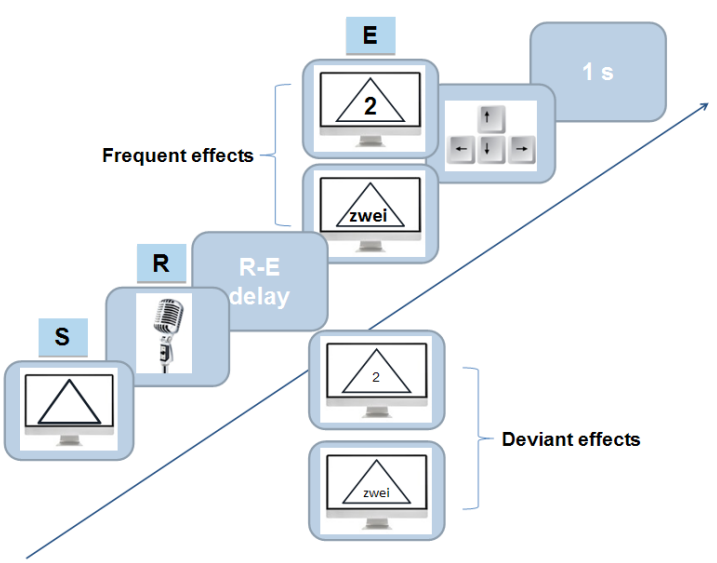

FIGURE 2.

Sequence of a trial in Experiment 1.

\section{PROCEDURE}

Participants were instructed to respond vocally to visual stimuli: They had to respond saying the word $z$ wei whenever they saw a triangle and saying the word acht whenever they saw a square (counterbalanced across participants). The vocal response triggered a visual R-E (i.e., the digit 2 or 8 in Arabic digit format or the corresponding number word), which was either compatible or incompatible to the response. The effect appeared $100 \mathrm{~ms}$ after speech onset and was visible for $500 \mathrm{~ms}$. The next trial started after $1 \mathrm{~s}$ of blank screen (see Figure 2).

Participants practiced the task in 12 trials without R-Es, which was followed by four test blocks, where both R-E compatibility conditions (compatible vs. incompatible) and effect format conditions (visual-symbolic vs. visual-verbal) were blocked. Compatible and incompatible conditions were always presented pairwise for both effect formats; condition orders were counterbalanced across participants (e.g., compatible-digit, incompatible-digit, compatible-number word, incompatible-number word).

Each of the four test blocks consisted of 160 trials. In $80 \%$ of the trials, the presented effect's size was relatively large, in which case participants were instructed to press the right arrow key after their vocal response. In $20 \%$ of the trials, however, the presented effect's size was relatively smaller, and in this case pressing of the down arrow key was required. In the instruction following the practice block, examples of the frequent and the deviant effects' size were displayed to the participants. The variation of effect size was included to attract participants' attention to processing the response effects, which were otherwise completely irrelevant to the instructed S-R task.

\section{TABLE 1.}

Examples for Effect Presentation in the Experimental Conditions

\begin{tabular}{ccc} 
& R-E compatible & R-E incompatible \\
\hline R-E code compatible & Two & Eight \\
R-E code incompatible & 2 & 8 \\
\hline
\end{tabular}

Note. The example depicts the visual effect presented following the vocal response "two" in R-E compatible/incompatible and R-E code compatible/incompatible conditions.

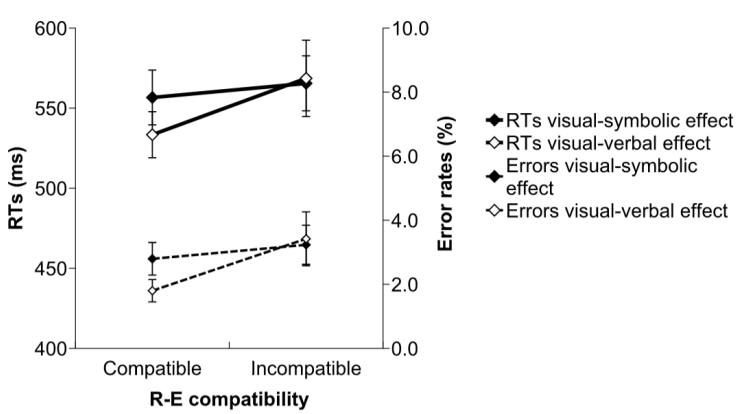

FIGURE 3.

Reaction Times (RTs; in milliseconds) and error rates (in \%) of Experiment 1 as a function of response-effect (R-E) compatibility and R-E code mapping (digit vs. number word). Error bars depict standard error of means.

\section{DESIGN}

The independent within-subject variables were R-E Compatibility 2) and Effect Code (2, see Table 1). Both independent variables were varied in a blockwise manner. The dependent variables were RT and Error Rate.

\section{Results}

RTs below $100 \mathrm{~ms}$ ( $1 \%$ of all trials) were discarded from the analysis assuming that these were due to technical voice-key irregularities (such as coughs, keypress noises, etc.). Further exclusions were made concerning the first trial of each condition, errors (2.8\%), trials after errors (2.3\%), trials after effect-deviant trials (20\%), and RTs with more than 2 SDs above or below each participant's mean (3.7\%). In total, $72.6 \%$ of the data were analyzed. Results are reported as significant at an alpha level of $p=.05$.

We submitted the mean RT data (see Figure 3) to a $2 \times 2$ (R-E Compatibility [compatible, incompatible] $\times$ Effect Code [visualsymbolic, visual-verbal]) analysis of variance (ANOVA). The ANOVA revealed a significant main effect of R-E compatibility, $F(1,23)=4.628$, $p=.042, \eta_{\mathrm{p}}{ }^{2}=.168$, reflecting RTs shorter by $22 \mathrm{~ms}$ in the compatible condition than in the incompatible condition (545 ms vs. $567 \mathrm{~ms}$ ). The main effect of effect code was not significant, $F<1$. There was a nonsignificant trend towards an interaction, $F(1,23)=2.696, p=.114$, $\eta_{\mathrm{p}}{ }^{2}=.105$, showing that the R-E compatibility effect tended to be more pronounced with visual-verbal effect code (i.e., number words, $35 \mathrm{~ms}$ ) than with visual-symbolic effect code (i.e., digits, $9 \mathrm{~ms}$; see Figure 3). While according to a post-hoc analysis, the $35 \mathrm{~ms}$ REC effect in the visual-verbal effect code condition was significant, $t(23)=2.517, p=$ .019 , the $9 \mathrm{~ms}$ REC effect was not significant in the digit condition, $t(23)=0.739, p=.467$. Further post-hoc tests show an effect of code compatibility only in R-E compatible trials, $t(23)=2.163, p=.041$, in RTs and $t(23)=3.180, p=.004$, in errors, while there is no such influence in R-E incompatible trials ( $p>.7$ for both RTs and errors).

Error rates (see Figure 3) were analyzed in a comparable $2 \times 2$ ANOVA which showed the same pattern, with significantly less errors in the R-E compatible condition (2.3\% vs. $3.3 \%), F(1,23)=5.616, p=$ $.027, \eta_{\mathrm{p}}{ }^{2}=.196$. There was a nonsignificant trend toward a main effect of 


\section{Method}

effect code, $F(1,23)=2.708, p=.113, \eta_{\mathrm{p}}{ }^{2}=.105$, and, like for RTs, also a nonsignificant trend toward an interaction between R-E Compatibility and Effect Code, $F(1,23)=2.837, p=.106, \eta_{p}^{2}=.110$, indicating that the REC effect tended to be larger with visual-verbal effect codes than with visual-symbolic effect codes (1.6\% vs. $0.4 \%$ ).

\section{Discussion}

Using the REC paradigm, Experiment 1 was conducted to explore a form of modality compatibility between effect formats that we term R-E code compatibility; specifically, we compared performance with visual-symbolic and visual-verbal response effects (i.e., digit vs. number word), keeping the vocal response modality constant. This way, we manipulated a specific form of modality compatibility that refers to the match of the central code of the response (vocal-verbal) and central code of the effect (visual-symbolic vs. visual-verbal).

We found an overall R-E compatibility benefit, which was numerically (but not statistically) more pronounced when the effect was R-E code compatible (i.e., a number word activating verbal codes). This pattern was similarly observed in both RT and error rates.

Since number words activate verbal codes while digits are visually presented symbols that activate a spatial code (Dehaene, 1997), the effect including a verbal code matches better with the verbal code of the vocal response modality of the task (Wickens \& Liu, 1988; Wickens et al., 1983). Thus, we assume that the trend for a more pronounced compatibility benefit in the number word condition was due to the compatibility between the response modality and the effect code (R-E code compatibility). The post-hoc observation showing an influence of (further) code compatibility in R-E compatible trials suggests that a perceptual overlap with respect to the verbal format is mainly effective when there is dimensional overlap as compared to R-E incompatible conditions in which (due to the reduced dimensional overlap) perceptual overlap does not play a role. However, the evidence for code-specific effects is not very strong in Experiment 1 . To examine the role of modality compatibility in R-E compatibility more directly, in Experiment 2, we compared performance with visual-verbal effect codes (as in Experiment 1) with that with auditory-verbal effect codes while vocal response modality was kept constant.

\section{EXPERIMENT 2}

Experiment 2 contrasted performance of a group which received auditory-verbal effects (i.e., spoken number words) with performance of another group which received visual-verbal effects (i.e., written number words). Both conditions are R-E code compatible, but according to the notion of modality compatibility, the vocal-auditory (i.e., modality compatible) R-E modality mapping condition should result in a larger REC benefit than in the vocal-visual (i.e., modality incompatible) condition ${ }^{1}$.

\section{PARTICIPANTS}

Forty-eight students (13 male, 36 female, two left-handed, $M_{\text {age }}$ $=24.89$ years) were recruited from RWTH Aachen University in the same manner as in Experiment 1. The participants were evenly distributed among the two modality compatibility groups, which were tested one after the other.

\section{APPARATUS AND PROCEDURE}

Participants were instructed to respond vocally to visually presented stimuli in the same manner as in Experiment 1. In the modality compatible condition, after their response, half of the participants heard the voice of a woman saying the effect (i.e., zwei or acht) in $80 \%$ of the trials (frequent effect) and the voice of a man in the remaining $20 \%$ of the trials (deviant effect; with gender of deviant effect type being counterbalanced across participants). The average duration of the auditory stimuli (used as R-Es) was $755 \mathrm{~ms}$ (female voice: $660 \mathrm{~ms}$; male voice: $850-900 \mathrm{~ms})^{2}$. In contrast, in the modality incompatible condition, visual effects were used, but unlike in Experiment 1, here we used solely number words, with the same parameters as in Experiment 1.

While the R-E delay in Experiment 1 was 100 ms, in Experiment 2, a longer R-E delay was introduced, giving enough time for the participants to respond before hearing or seeing the effect. Using auditory effects, pilot studies suggested that the $100 \mathrm{~ms}$ delay was too short, since the participants were almost always still responding when the effect was presented, so that the longer R-E delay was necessary to make sure that the auditory effect was not presented while the participant was still responding. For exploratory reasons, we included two different R-E delays ( $300 \mathrm{~ms}$ vs. $400 \mathrm{~ms}$ ) in a blocked manner ${ }^{3}$. The next trial started after $1 \mathrm{~s}$ of blank screen.

The instruction about the S-R mapping was like in Experiment 1, but in Experiment 2, participants had to register only the deviant trials by pressing the down arrow key on the keyboard.

Twelve practice trials familiarized the participants with the task, followed by four experimental blocks of 160 trials each. In these, both compatible and incompatible conditions as well as the R-E delay conditions were blocked.

\section{DESIGN}

The independent variables were R-E Compatibility (2; within-subject) and R-E Modality (2; between-subjects). The dependent variables were RT and Error Rates.

\section{Results}

The same exclusion criteria were applied to the analysis of Experiment 2 as in Experiment 1. Errors (1.4\%), trials after errors (1.2\%), RTs shorter than $100 \mathrm{~ms}(1.2 \%)$ and more than 2 SDs (3.3\%) above or below each participant's mean, as well as trials after deviant trials (20\%) were discarded from the analysis. In total, $74.9 \%$ of the raw data was analyzed. 


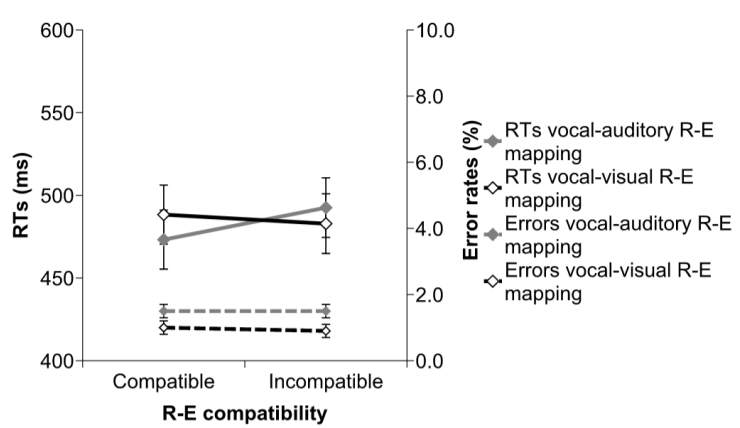

FIGURE 4.

Reaction Times (RTs; in milliseconds) and error rates (in \%) in Experiment 2 as a function of response-effect (R-E) compatibility and R-E modality mapping (vocal-auditory vs. vocal-visual). Error bars depict standard error of means.

We submitted the RT data to a $2 \times 2$ mixed ANOVA (R-E Compatibility [compatible, incompatible] $\times$ R-E Modality [vocal-auditory, vocal-visual; between-subjects]). The ANOVA revealed a nonsignificant trend toward a main effect of R-E compatibility, $F(1,46)=$ $2.912, p=.095, \eta_{\mathrm{p}}{ }^{2}=.060$. The effect of the between-subjects variable (R-E Modality) was not significant $(F<1.00)$, but a significant interaction was found between R-E Compatibility and R-E Modality, $F(1,46)$ $=9.339, p=.004, \eta_{\mathrm{p}}^{2}=.169$ (see Figure 4 ). Follow-up tests showed that the $19 \mathrm{~ms}$ REC effect found in the vocal-auditory condition (compatible R-E modality mapping) was significant, $t(23)=-3.308, p=.003$, whereas the slightly reversed effect $(-6 \mathrm{~ms})$ with the incompatible (vocal-visual) R-E modality mapping was not significant, $t(23)=.972$, $p>.341$. The analysis of errors did not show any significant effect, Fs $<1.00$; see Figure 4 .

\section{Discussion}

Experiment 2 was designed to investigate R-E modality compatibility in the REC paradigm, comparing directly the influence of auditory versus visual effect modality while keeping the response modality constant (vocal). As predicted, the REC effect was stronger with vocalauditory modality mapping than with vocal-visual R-E mapping. In fact, the REC effect was found only with the compatible R-E modality mapping (i.e., vocal-auditory), suggesting that participants anticipated their actions' effects, whereas the REC effect was not significant in the incompatible R-E modality mapping condition (i.e., vocal-visual). That is, the present evidence supports the hypothesis that REC effects are larger with modality compatible R-E mappings if tested against fully comparable but modality-incompatible R-E mappings. The fact that the REC effect with the vocal-visual R-E mapping was clearly significant in Experiment 1 but not in Experiment 2 might be due to the lengthened R-E delay in Experiment 2 relative to Experiment 1. The issue of a potential influence of the R-E delay on the REC effect may be interesting in itself, but it should not distract from the fact that, under comparable conditions, the REC effect was significantly larger with the modality-compatible R-E mapping (see Janczyk, Durst, \& Ulrich, 2016, for a discussion of the role of R-E delay on the REC effect).

\section{GENERAL DISCUSSION}

The influence of modality compatibility has already been investigated in multitasking conditions (Göthe et al., 2016; Greenwald, 1972; Hazeltine et al., 2006; Stephan \& Koch, 2010, 2011). In the present study, two experiments aimed to explore the role of modality compatibility in an R-E task, introducing the notion of modality-specificity into research on action control based on ideomotor theory (Badets et al., 2016; Greenwald, 1970; Shin et al., 2010).

In Experiment 1, we used vocal responses and visual effects, varying the type of effect code to compare performance with visualverbal (number words) and with visual-symbolic (digits) central effect codes (Göthe et al., 2016; Wickens \& Liu, 1988; Wickens et al., 1983). According to Wickens and Liu (1988), performance can be predicted based on a model of three components: input modality (visual or auditory), central codes (verbal or spatial), and output modality (manual or vocal). Performance is predicted to be better if the components match with each other within a task (Göthe et al., 2016; Wickens et al., 1983). This view is close to the notion of modality compatibility, since both suggest that a certain mapping between input and output modalities is more optimal than other mappings. Thus, based on Wickens and Liu's view, we expected to find a larger REC benefit in the verbal number word effect condition compared to the spatial digit effect condition, reflecting R-E code compatibility. The results revealed an overall REC effect, and indeed, this effect was more pronounced when the effect code was a number word rather than when it was a digit. Note that even though this effect was present only at the trend level, it was so in both RTs $(p=.114)$ and error rates $(p=.106)$, which increases our confidence that it represents a real, albeit small effect.

In Experiment 2, we manipulated the effect modality directly so that the vocal response was followed either by an auditory-verbal response effect (i.e., spoken number word) or by a visual-verbal response effect (i.e., written number word). This way, we could compare performance with a modality compatible R-E mapping and with a modality incompatible R-E mapping. The results indeed showed a significantly larger REC effect when the R-E modality mapping was compatible than when it was incompatible.

Compared to the evidence for a role of R-E modality compatibility, there is somewhat less suggestive and statistically weaker evidence for an influence of R-E code compatibility on the size of the REC effect. At the same time, empirical findings are stronger in favor of the hypothesis that the modality-specific nature of the R-E relations plays a role in addition to more abstract, conceptual dimensional overlap relations (e.g., ideomotor compatibility; Greenwald, 1972). 
The results of Experiment 2 fit well with previous findings suggesting evidence for an influence of modality compatibility in S-R tasks in different paradigms (dual task; see, e.g., Göthe et al., 2016; Greenwald, 1972; Hazeltine et al., 2006; task switching; see, e.g., Stephan \& Koch, 2010, 2011, 2016). Interestingly, in our REC experiments, which use single-task conditions, we observed that the REC effect disappeared with the modality incompatible R-E mapping under some conditions, while the influence of modality in multitasking settings has proven to be very robust. This may be an important finding, considering that previous studies on multitasking also found an effect of (S-R) modality compatibility, primarily in mixed-task conditions, but very rarely so in single-task conditions (e.g., Lukas et al., 2010; Schäffner et al., 2016; Stephan \& Koch, 2010, 2016). Therefore, given this pattern of results within our study and across other published studies, we speculate that modality compatibility is an even more influential factor determining crosstalk in conditions in which there is uncertainty (i.e., variability, such as in task switching studies) about modality mappings. Future research should be extended to such experimental designs where, due to this uncertainty, the cognitive load is higher (e.g., task switching with R-Es).

Finally, while our goal was to investigate R-E code compatibility and R-E modality compatibility, we are aware that the present study was limited to the comparison of only two R-E modality mappings using vocal responses. Thus, future experiments would need to explore the nature of R-E relations also in tasks involving different responses, such as using manual responses, and different types of effects, such as tactile effects, in order to examine modality compatibility in a more exhaustive manner.

\section{FOOTNOTES}

${ }^{1}$ The condition labeled as visual-verbal effect code condition in Experiment 1, will be referred to as vocal-visual R-E modality mapping in Experiment 2. This difference in terminology is made to highlight the relevant manipulation in the experiments.

${ }^{2}$ The analysis did not reveal any influence of whether the effect was pronounced by a man or a woman.

${ }^{3}$ The delays' length was not significant as a main effect, $F(1,46)=$ $1.025, p=.317$. There was a non-significant trend toward an interaction of R-E delay length and R-E compatibility, $F(1,46)=2.740, p=.105$, indicating an overall larger REC effect with longer R-E delay. However, since the R-E delay itself was not the main focus of the present study and there were no further significant interactions involving the R-E delay, these results will not be further discussed. R-E delays have recently been investigated by Dignath and Janczyk (2016).

\section{AUTHOR NOTE}

This research was supported by the DFG (Deutsche Forschungsgemeinschaft, KO 2045/18-1).

\section{REFERENCES}

Ansorge, U. (2002). Spatial intention-response compatibility. Acta Psychologica 109, 285-299. doi: 10.1016/S0001-

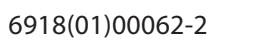

Badets, A., Koch, I., \& Philipp, A. M. (2016). A review of ideomotor approaches to perception, cognition, action, and language: Advancing a cultural recycling hypothesis. Psychological Research, 80, 1-15. doi: 10.1007/s00426-014-0643-8 [wW

Badets, A., Koch, I., \& Touissaint , L. (2013). Role of an ideomotor mechanism in number processing. Experimental Psychology, 60, 34-43. doi: 10.1027/1618-3169/a000171 |wWW

Badets, A., \& Pesenti, M. (2011). Finger-number interaction: An ideomotor account. Experimental Psychology, 58, 287-292. doi: 10.1027/1618-3169/a000095 (WWW

Dehaene, S. (1997). The number sense. New York, NY: Oxford University Press.

Dehaene, S., Bossini, S., \& Giraux, P. (1993). The mental representation of parity and number magnitude. Journal of Experimental Psychology: General, 122, 371-396. doi: 10.1037/0096-3445 .122.3.371

Dignath, D., \& Janczyk, M. (2016). Anticipation of delayed actioneffects: learning when an effect occurs, without knowing what this effect will be. Psychological Research. Advance online publication. doi: 10.1007/s00426-016-0797-7 WWW

Elsner, B., \& Hommel, B. (2001). Effect anticipation and action control. Journal of Experimental Psychology: Human Perception and Performance, 27, 229-240. doi: 10.1037/0096-1523.27.1.229 WWW

Göthe, K., Oberauer, K., \& Kliegl, R. (2016). Eliminating dualtask costs by minimizing crosstalk between tasks: The role of modality and feature pairings. Cognition, 150, 92-108. doi: 10.1016/j.cognition.2016.02.003 [WWW

Greenwald, A. G. (1970). Sensory feedback mechanisms in performance control: With special reference to the ideomotor mechanism. Psychological Review, 77, 73-99. doi: 10.1037/ h0028689

Greenwald, A. G. (1972). On doing two things at once: Time sharing as a function of ideomotor compatibility. Journal of Experimental Psychology, 94, 52-57. | $\overline{\mathrm{WWW}}$

Greenwald, A. G. (2005). A reminder about procedures needed to reliably produce perfect timesharing: Comment on Lien, McCann, Ruthruff, and Proctor (2005). Journal of Experimental Psychology: Human Perception and Performance, 31, 221-225. doi: 10.1037/0096-1523.31.1.221 |WWW

Greenwald, A. G., \& Shulman, H. G. (1973). On doing two things at once: II. Elimination of the psychological refractory period effect. Journal of Experimental Psychology, 101, 70-76. doi: $10.1037 / \mathrm{h} 0035451$ WWW

Hazeltine, E., Ruthruff, E., \& Remington, R. W. (2006). The role of input and output modality pairings in dual-task performance: Evidence for content-dependent central interference. Cognitive Psychology, 52, 291-435. doi: 10.1016/j.cogpsych.2005.11.001 WWW

Herwig, A., Prinz, W., \& Waszak, F. (2007). Two modes of sensorimotor integration in intention-based and stimulus-based actions. Quarterly Journal of Experimental Psychology, 60, 1540-

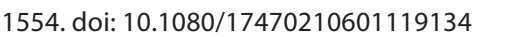


Herwig, A., \& Waszak, F. (2009). Intention and attention in ideomotor learning. Quarterly Journal of Experimental Psychology, 62, 219-227. doi: 10.1080/17470210802373290 WWW

Hommel, B. (2013). Ideomotor action control: On the perceptual grounding of voluntary actions and agents. In W. Prinz, M. Beisert, \& A. Herwig (Eds.), Action science: Foundations of an emerging discipline (pp. 113-136). Cambridge, MA: MIT Press.

Hommel, B., Alonso, D., \& Fuentes, L. J. (2003). Acquisition and generalization of action effects. Visual Cognition, 10, 965-986. doi: 10.1080/13506280344000176

Janczyk, M., Durst, M., \& Ulrich, R. (2016). Action selection by temporally distal goal-states. Psychonomic Bulletin and Review. Advance online publication. doi: 10.3758/s13423-016-1096-4 WWW

Janczyk, M., Heinemann, A., \& Pfister, R. (2012). Instant attraction: Immediate action-effect bindings occur for both, stimulusand goal-driven actions. Frontiers in Psychology, 3:446. doi: 10.3389/fpsyg.2012.00446 WWW

Janczyk, M., Pfister, R., \& Kunde, W. (2012). On the persistence of tool-based compatibility effects. Journal of Psychology, 220, 1622. doi: 10.1027/2151-2604/a000086

Janczyk, M., Yamaguchi, M., Proctor, R.W., \& Pfister, R. (2015). Response-effect compatibility with complex actions: The case of wheel rotations. Attention, Perception, \& Psychophysics, 77, 930-940. doi: 10.3758/s13414-014-0828-7 |WWW

Keller, P. E., \& Koch, I. (2006). Exogenous and endogenous response priming with auditory stimuli. Advances in Cognitive Psychology, 2, 269-276. doi: 10.2478/v10053-008-0061-9

Koch, I., Keller, P., \& Prinz, W (2004). The ideomotor approach to action control: Implications for skilled performance. International Journal of Sport and Excercise Psychology, 2, 362375. doi: 10.1080/1612197X.2004.9671751

Koch, I., \& Kunde, W. (2002). Verbal response-effect compatibility. Memory \& Cognition, 30, 1297-1303. doi: 10.3758/BF03213411

Kornblum, S., Hasbroucq, T., \& Osman, A. (1990). Dimensional overlap: Cognitive basis for stimulus-response compatibility: A model and taxonomy. Psychological Review, 97, 253-270. doi: 10.1037/0033-295X.97.2.253 $\underline{\underline{\mathrm{WWW}}}$

Kunde, W. (2001). Response-effect compatibility in manual choice reaction tasks. Journal of Experimental Psychology: Human Perception and Performance, 27, 387-394. doi: 10.1037/00961523.27.2.387 |WWW

Kunde, W., Koch, I., \& Hoffmann, J. (2004). Anticipated action effects affect the selection, initiation and execution of actions. Quarterly Journal of Experimental Psychology, 57A, 87-106. doi: 10.1080/02724980343000143 $\overline{\mathrm{WWW}}$

Lien, M. C., Proctor, R. W., \& Allen, P. A. (2002). Ideomotor compatibility in the psychological refractory period effect: 29 years of oversimplification. Journal of Experimental Psychology: Human Perception and Performance, 28, 396-409. doi: 10.1037/00961523.28.2.396 $\overline{\text { WWW }}$

Lukas, S., Philipp, M. A., \& Koch, I. (2010). The role of preparation and cue-modality crossmodal task switching. ActaPsychologica, 134, 318-322. doi: 10.1016/j.actpsy.2010.03.004 WWW

Pfister, R., Kiesel, A., Hoffmann, J. (2011). Learning at any rate: Action-effect learning for stimulus based actions. Psychological Research, 75, 61-65. doi: 10.1007/s00426-010-0288-1 |WWW

Pfister, R., Kiesel, A., \& Melcher, T. (2010). Adaptive control of ideomotor effect anticipation. Acta Psychologica, 135, 316-322. doi: 10.1016/j.actpsy.2010.08.006 WWW

Pfister, R., \& Kunde, W. (2013). Dissecting the response in response-effect compatibility. Experimental Brain Research, 224, 647-655. doi: 10.1007/s00221-012-3343-x WWW

Schäffner, S., Koch, I., \& Philipp, A. M. (2016). The role of sensory-motor modality compatibility in language processing. Psychological Research, 80, 212-223. doi: 10.1007/s00426-015 $-0661-1 \underline{\underline{W W}}$

Shin, Y. K., Proctor, R. W., \& Capaldi, E. J. (2010). A review of contemporary ideomotor theory. Psychological Bulletin, 136, 943947. doi: 10.1037/a0020541 WWW

Stephan, D. N., \& Koch, I. (2010). Central crosstalk in task switching: Evidence from manipulating input-output modality compatibility. Journal of Experimental Psychology: Learning, Memory \& Cognition, 36, 1075-1081. doi: 10.1037/a0019695 |www|

Stephan, D. N., \& Koch, I. (2011). The role of input-output modality compatibility in task swtiching. Psychological Research, 75, 491-498. doi: 10.1007/s00426-011-0353-4 WWW

Stephan, D. N., \& Koch, I. (2016). Modality-specific effects on crosstalk in task switching: Evidence from modality compatibility. Psychological Research, 80, 935-943. doi: 10.1007/

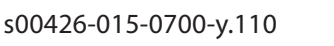

Walsh, V. (2003). A theory of magnitude: Common cortical metrics of time, space and quantity. Trends in Cognitive Sciences, 7, 483-488. doi: 10.1016/j.tics.2003.09.002 WWW

Wickens, C. D., \& Liu, Y. (1988). Codes and modalities in multiple resources: A success and a qualification. Human Factors, 30, 599-616. doi: 10.1177/001872088803000505 WWW

Wickens, C. D., Sandry, D. L., \& Vidulich, M. (1983). Compatibility and resource competition between modalities of input, central processing and output. Human Factors, 25, 227-248. doi: 10.1177/001872088302500209 WWW

Ziessler, M., Nattkemper, D., \& Frensch, P. A. (2004). The role of anticipation and intention for the learning of effects of selfperformed actions. Psychological Research, 68, 163-175. doi: 10.1007/s00426-003-0153-6 WWW

RECEIVED 25.05.2016 | ACCEPTED 03.02.2017 